\title{
Facile Preparation of 2-Arylbenzo[b]furan Molecules and Their Anti-inflammatory Effects
}

\author{
Jung Woon Hwang, ${ }^{\dagger}$ Da Hye Choi, ${ }^{\dagger}$ Jae-Ho Jeon, ${ }^{\ddagger}$ Jin-Kyung Kim, ${ }^{\S}$ and Jong-Gab Jun ${ }^{\dagger},,, *$ \\ ${ }^{\dagger}$ Department of Chemistry and Institute of Applied Chemistry, ${ }^{\dagger}$ Institute of Natural Medicine, \\ and ${ }^{\S}$ Center for Efficacy Assessment and Development of Functional Foods and Drugs, Hallym University, \\ Chuncheon 200-702, Korea.*E-mail: jgjun@hallym.ac.kr \\ Received November 25, 2009, Accepted March 2, 2010
}

\begin{abstract}
An efficient and practical preparation of 2-arylbenzo[b]furan molecules including natural egonol, XH-14, ailanthoidol, and unnatural derivatives is demonstrated using Sonogashira coupling, iodine induced cyclization and Wittig reaction. Anti-inflammatory effects of the prepared benzo $[b]$ furans were examined in lipopolysaccharide (LPS)-stimulated RAW 264-7 macrophages. The results showed that ailanthoidol, XH-14 and three other unnatural derivatives $(\mathbf{9 - 1 0}, 13)$ inhibited significantly the production of inflammatory mediator nitric oxide without showing cytotoxicity.
\end{abstract}

Key Words: Ailanthoidol, XH-14, 2-Arylbenzo[b]furan, Anti-inflammatory, Nitric oxide

\section{Introduction}

The 2-arylbenzo[b]furan structure is prevalent in a wide variety of biologically active natural and unnatural compounds. ${ }^{1}$ Many 2-arylbenzo $[b]$ furan derivatives are well-known to exhibit broad range of biological activities including anticancer, ${ }^{2}$ antiproliferative, ${ }^{3}$ anti-inflammatory, ${ }^{4}$ antiviral, ${ }^{5}$ antifungal, ${ }^{6}$ immunosuppressive, ${ }^{7}$ antiplatelet, ${ }^{8}$ antioxidative, ${ }^{9}$ antifeedent, ${ }^{10}$ and insecticidal activities. ${ }^{11}$ The investigation of structure-activity relationships for 2-arylbenzo[b]furan substituents is still attractive due to variety of biological activities. A number of synthetic approaches to the 2-arylbenzo[ $b]$ furan derivatives have been introduced in recent years. ${ }^{12}$ Recently, we synthesized ego- nol 2, isolated from the seed oil of Styrax japonicum, in 5 step reaction procedures in $74 \%$ overall yield from vanillin $\mathbf{1}$, and also prepared its derivatives 3-7 (Fig. 1). ${ }^{13}$

Another 2-arylbenzo[b]furan natural product XH-14 (8, Fig. 2), isolated from Salvia miltiorrhiza Bunge (Chinese name "Danshen") which has been widely used in China for the treatment of cardiovascular diseases such as acute myocardial infarction and angina pectoris, ${ }^{14}$ was also synthesized in 9 steps overall $23 \%$ yield from vanillin by mainly using Sonogashira coupling reaction with iodine induced cyclization. ${ }^{15}$ The key features of our synthesis of benzofuran nucleus were regioselective halogenation, Sonogashira coupling and halogen-induced cyclization in different substituents including optimiza-<smiles>COc1cc(C=O)ccc1O</smiles>

Vanillin (1)

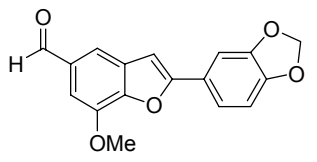

5<smiles>COc1cc(CCCO)cc2cc(-c3ccc4c(c3)OCO4)oc12</smiles>

Egonol (2)<smiles>COc1cc(CCCOC(C)=O)cc2cc(-c3ccc4c(c3)OCO4)oc12</smiles>

3<smiles>CCOC(=O)/C=C/c1cc(OC)c2oc(-c3ccc4c(c3)OCO4)cc2c1</smiles>

6<smiles></smiles>

4<smiles>COc1cc(/C=C/CO)cc2cc(-c3ccc4c(c3)OCO4)oc12</smiles>

7<smiles>COc1cc(-c2oc3cc(CCCO)c(OC)cc3c2C=O)ccc1O</smiles>

10

Figure 2. Structures of $\mathrm{XH}-14$ and derivatives. 
<smiles>COc1cc(-c2cc3cc(CCCO)cc(OC)c3o2)ccc1O</smiles>

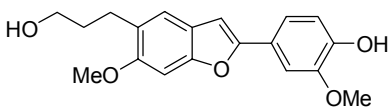<smiles>COc1cc(-c2cc3cc(OC)c(CCCO)cc3o2)ccc1O</smiles>

13

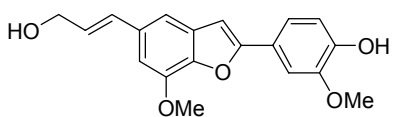

Ailanthoidol (14)

Figure 3. Structures of 3-deformylated 2-arylbenzo[b]furans.

tion of the synthetic sequences. The modifications of XH-14 were given only to the synthesis of C-2 and C-3 substituted analogs. Due to its high selectivity for the $\mathrm{A}_{1}$ receptor subtype, the preparation of analogs for SAR tests was clearly of interest. In order to prove the role of other substituents on XH-14 in biological selectivity, the derivatives of $\mathrm{XH}-14,9$ and 10, were prepared using similar procedures in 9 steps overall $30 \%$ and $55 \%$ yield, respectively. ${ }^{16}$ In this report, we describe the synthesis of 3-deformylated benzofurans 11-14 (Fig. 3) including ailanthoidol 14 and the comparison of anti-inflammatory effects for the prepared 13 benzofurans 2-14.

\section{Results and Discussion}

In order to prove the importance of 3-formyl substituent in 2-arylbenzo[b]furans for their biological activities, we prepared 3-deformylated derivatives of XH-14 (8) and analogues 9-10. The synthetic intermediates 15-17, ${ }^{15,16}$ which have 3-iodo substituent, for the syntheses of XH-14 and analogues 9-10 were easily transformed to deiodo-benzofurans 18-20 by using $\mathrm{LiAlH}_{4}$ reduction, and which were then debenzylated with $\mathrm{BBr}_{3}$ to afford the desired 3-deformylated benzofurans 11-13 in high yields (Scheme 1).

The ailanthoidol 14, which is a natural 3-deformylated 2-arylbenzo $[b]$ furan, was isolated from the chloroform-soluble fraction of the tree of Zanthoxylum ailanthoidos. ${ }^{17}$ While there have been no reports on this compound's biological activities, extracts of the bark and leaves of this tree have been used in folk medicine.

Previous synthetic strategies for $\mathrm{XH}-14$, basically, were applied for the ailanthoidol synthesis as shown in Scheme 2. Regioselective iodination of vanillin 1 using $\mathrm{I}_{2}-\mathrm{Ag}_{2} \mathrm{SO}_{4}$ in $\mathrm{EtOH}$ at rt gave 3-iodovanillin 21 in $85 \%$ yield. Sonogashira coupling reaction of the iodovanillin with the acetylene 22 by using $\mathrm{Pd}\left(\mathrm{PPh}_{3}\right)_{4} / \mathrm{CuI} / \mathrm{TEA} / \mathrm{DMF}$ resulted in a coupled and cyclized benzofuran product 23 in $92 \%$ yield. Direct cyclization of the coupled product in one-flask is due to the presence of free 4-OH group of iodovanillin $\mathbf{2 1}$. The only coupled product without cyclization can be isolated with the protection of the 4-OH of iodovanillin in the reaction. The Wittig reaction of the coupled benzofuran 23 in methylene chloride at reflux with (carbethoxymethylene)triphenylphosphorane produced $99 \%$ yield of only (E)-24 which was then debenzylated to $\mathbf{2 5}(98 \%)$ with $\mathrm{TiCl}_{4}$ and reduced to ailanthoidol 14 (95\%) using DIBAL-H.
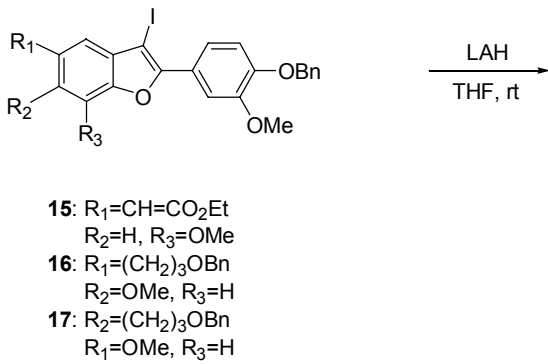<smiles>[R]c1cc2cc(-c3ccc(OC)c(OC)c3)oc2c([R])c1[R]</smiles>

18: $\mathrm{R}_{1}=\left(\mathrm{CH}_{2}\right)_{3} \mathrm{OH}$ $\mathrm{R}_{2}=\mathrm{H}, \mathrm{R}_{3}=\mathrm{OMe}$ 19: $\mathrm{R}_{1}=\left(\mathrm{CH}_{2}\right)_{3} \mathrm{OBn}$ $\mathrm{R}_{2}=\mathrm{OMe}, \mathrm{R}_{3}=\mathrm{H}$ 20: $\mathrm{R}_{2}=\left(\mathrm{CH}_{2}\right)_{3} \mathrm{OBn}$ $\mathrm{R}_{1}=\mathrm{OMe}, \mathrm{R}_{3}=\mathrm{H}$

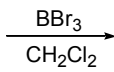<smiles>[R]c1cc2cc(-c3ccc(O)c(OC)c3)oc2c([R3])c1[R2]</smiles>

$$
\text { 11: } \begin{aligned}
\mathrm{R}_{1} & =\left(\mathrm{CH}_{2}\right)_{3} \mathrm{OH} \\
\mathrm{R}_{2} & =\mathrm{H}, \mathrm{R}_{3}=\mathrm{OMe} \\
\text { 12: } \mathrm{R}_{1} & =\left(\mathrm{CH}_{2}\right)_{3} \mathrm{OH} \\
\mathrm{R}_{2} & =\mathrm{OMe}, \mathrm{R}_{3}=\mathrm{H} \\
\text { 13: } \mathrm{R}_{2} & =\left(\mathrm{CH}_{2}\right)_{3} \mathrm{OH} \\
\mathrm{R}_{1} & =\mathrm{OMe}, \mathrm{R}_{3}=\mathrm{H}
\end{aligned}
$$

Scheme 1. Syntheses of deformylated 2-arylbenzo[b]furans 11-13<smiles>COc1cc(C=O)ccc1O</smiles>

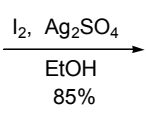

1<smiles>CCOC(=O)/C=C/c1cc(OC)c2oc(-c3ccc(OCc4ccccc4)c(OC)c3)cc2c1</smiles>

24<smiles>O=Cc1cc(O)c(O)c(I)c1</smiles>

21<smiles>CCOC(=O)/C=C/c1cc(OC)c2oc(-c3ccc(O)c(OC)c3)cc2c1</smiles>

25

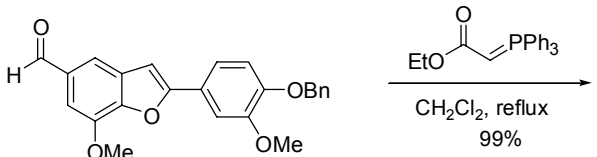

23

Scheme 2. Total synthesis of ailanthoidol 14 from vanillin 1 
(a)

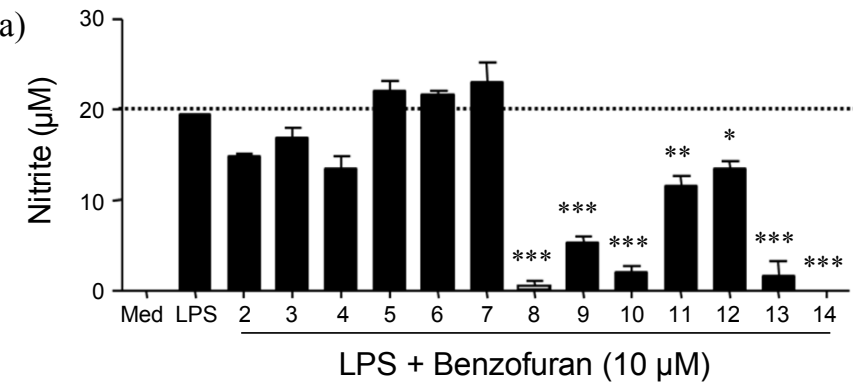

(b)

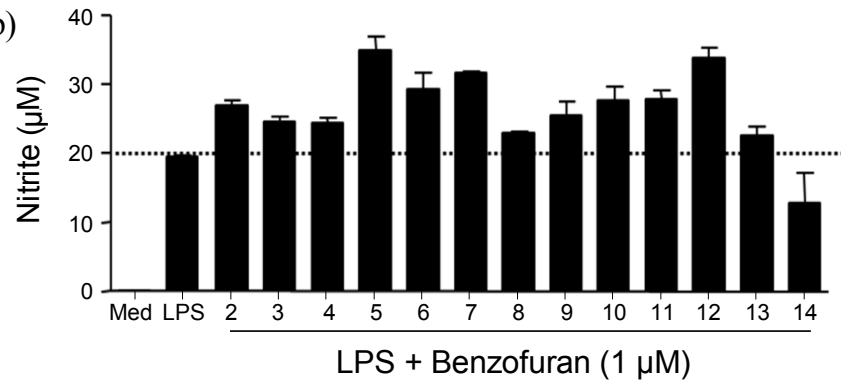

Figure 4. Effects of 2-arylbenzo[b]furans 2-14 on LPS-induced NO production. RAW264.7 cells were treated with (a) $10 \mu \mathrm{M}$ and (b) $1 \mu \mathrm{M}$ of benzofurans in the presence of $1 \mu \mathrm{g} \mathrm{mL} \mathrm{m}^{-1}$ of LPS, and NO production was determined. Statistical significance is based on the difference when compared with LPS-stimulated cells $(* P<0.05, * * P<0.01, * * * P<0.001)$.

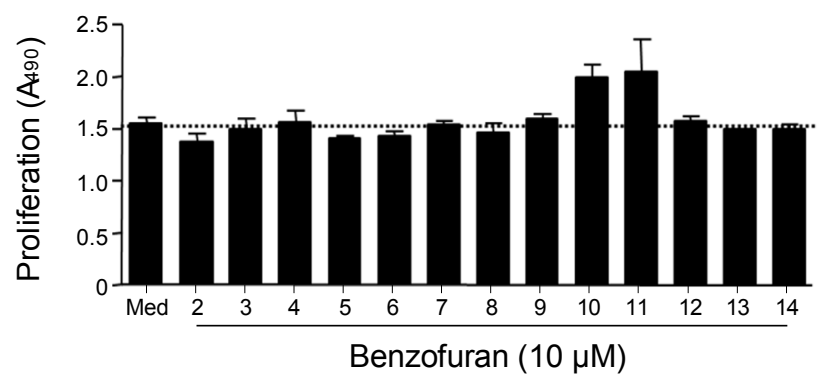

Figure 5. Cell viability assay of 2-arylbenzo[b]furans 2-14 at $10 \mu \mathrm{M}$.

Inflammation is a beneficial host response to a foreign challenge or tissue injury that leads ultimately to the restoration of normal tissue structure and function, however, prolonged inflammation contributes to the pathogenesis of many inflammatory diseases. ${ }^{18}$ In order to investigate the anti-inflammatory properties of the prepared 13 benzofurans $\mathbf{2 - 1 4}$, we measured the amount of nitric oxide (NO), which is one of the essential mediators on inflammation, in lipopolysaccharide (LPS)-stimulated RAW264.7 macrophages (Fig. 4 and Table 1). ${ }^{19}$ Benzofurans 8-14 (Fig. 4a, $10 \mu \mathrm{M}$ ) significantly suppressed the production of NO in LPS-stimulated RAW264.7 cells. The strong inhibitory activity was shown in benzofurans $8,10,13$ and 14. Among these active compounds, ailanthoidol 14 showed $100 \%$ inhibition of NO production at $10 \mu \mathrm{M}$ and $35 \%$ inhibition even at $1 \mu \mathrm{M}$ (Fig. 4b). As shown in Fig. 5, the cell viability was not affected by the all synthetic benzofurans 2-14, indicating no cytotoxicity.

In conclusion, the practical and optimized 5 step reaction procedures produced ailanthoidol (14) in $72 \%$ overall yield from vanillin. Ailanthoidol, egonol, XH-14 and their derivatives were examined their anti-inflammatory activity in lipopolysaccharide (LPS)-stimulated RAW 264-7 macrophages. Among these benzofurans, ailanthoidol showed $100 \%$ inhibition of NO production at $10 \mu \mathrm{M}$ and $35 \%$ inhibition even at $1 \mu \mathrm{M}$. The cell viability assay at $10 \mu \mathrm{M}$ indicated that the all synthetic benzofurans 2-14 did not show any significant cytotoxicity.

\section{Experimental}

All chemicals used were purchased from commercial sources and used as received unless otherwise stated. NMR spectra were recorded at Varian Mercury-300 MHz FT-NMR for ${ }^{1} \mathrm{H}$ and $75 \mathrm{MHz}$ for ${ }^{13} \mathrm{C}$, with the chemical shifts $(\delta)$ reported in parts per million (ppm) relative to TMS and the coupling con-

Table 1. Inhibitory activities of 2-arylbenzo[b]furans 2-14

Compound

NO production (\% inhibition)

\begin{tabular}{cc}
\hline $10 \mu \mathrm{M}$ & $1 \mu \mathrm{M}$ \\
$76.1 \pm 0.5(23.9)$ & $138.1 \pm 0.9(-38.1)$ \\
$86.9 \pm 1.4(13.1)$ & $126.3 \pm 1.1(-26.3)$ \\
$69.6 \pm 1.8(30.4)$ & $125.0 \pm 1.0(-25.0)$ \\
$113.4 \pm 1.5(-13.4)$ & $179.1 \pm 2.8(-79.1)$ \\
$110.9 \pm 0.7(-10.9)$ & $150.3 \pm 3.3(-50.3)$ \\
$118.3 \pm 3.1(-18.3)$ & $162.4 \pm 0.4(-62.4)$ \\
$2.7 \pm 0.7(97.3)$ & $117.1 \pm 0.3(-17.1)$ \\
$27.0 \pm 1.0(73.0)$ & $130.3 \pm 2.6(-30.3)$ \\
$10.3 \pm 1.0(89.7)$ & $142.0 \pm 2.5(-42.0)$ \\
$59.6 \pm 1.5(40.4)$ & $143.1 \pm 1.8(-43.1)$ \\
$69.0 \pm 1.1(31.0)$ & $173.4 \pm 2.2(-73.4)$ \\
$8.3 \pm 2.2(91.7)$ & $116.2 \pm 1.7(-16.2)$ \\
$0.0 \pm 0.0(\mathbf{1 0 0 . 0})$ & $65.1 \pm 6.1(\mathbf{3 4 . 9})$ \\
$100 \pm 0.2(0.0)$ & $100 \pm 0.2(0.0)$ \\
\hline
\end{tabular}

Egonol (2)

5-(3-Actyloxypropyl)-7-methoxy-2-(3,4-methylenedioxyphenyl)benzofuran (3)

5-(3-Propanoyloxypropyl)-7-methoxy-2-(3,4-methylenedioxyphenyl)benzofuran (4)

5-(3-Hydroxyprop-1-en-yl)-7-methoxy-2-(3,4-methylenedioxyphenyl)benzofuran (5)

5-Formyl-7-methoxy-2-(3,4-methylenedioxyphenyl)benzofuran (6)

5-Carbethoxyethenyl-7-methoxy-2-(3,4-methylenedioxyphenyl)benzofuran (7)

XH-14 (8)

2-(4-Hydroxy-3-methoxyphenyl)-5-(3-hydroxypropyl)-6-methoxybenzofuran-3-carbaldehyde (9)

2-(4-Hydroxy-3-methoxyphenyl)-6-(3-hydroxypropyl)-5-methoxybenzofuran-3-carbaldehyde (10)

2-(4-Hydroxy-3-methoxyphenyl)-5-(3-hydroxypropyl)-7-methoxybenzofuran (11)

2-(4-Hydroxy-3-methoxyphenyl)-5-(3-hydroxypropyl)-6-methoxybenzofurane (12)

2-(4-Hydroxy-3-methoxyphenyl)-5-(3-hydroxypropyl)-6-methoxybenzofurane (13)

Ailanthoidol (14)

LPS

The results are reported as mean value \pm SEM for $n=3 . \%$ inhibition is based on LPS as shown in parentheses. 
stants $(J)$ quoted in $\mathrm{Hz}$. $\mathrm{CDCl}_{3}$ was used as a solvent and an internal standard. Infrared spectra were recorded on a Shimadzu IR-435 spectrometer. GC-MS analyses were performed using a HP-5890/JMS-AM 150, JEOL. Flash chromatography was carried out using silica gel Merck 60 (230 - 400 mesh). Thinlayer chromatography (TLC) was performed on DC-Plastikfolien $60, \mathrm{~F}_{254}$ (Merck, layer thickness $0.2 \mathrm{~mm}$ ) plastic-backed silica gel plates with visualization by UV light $(254 \mathrm{~nm})$ or by treatment with $p$-anisaldehyde. Melting points were measured on a MEL-TEMP II apparatus and were uncorrected. LPS derived from Escherichia coli was obtained from Sigma (St Louis, Mo, USA). The Dulbecco's modified Eagle's medium (DMEM), fetal bovine serum (FBS), penicillin, and streptomycin used in this study were obtained from Hyclone (Logan, Utah, USA).

\section{General procedure of deiodination.}

2-(4-Benzyloxy-3-methoxyphenyl)-5-(3-hydroxypropyl)-7methoxybenzofuran (18): 2-(4-Benzyloxy-3-methoxyphenyl)5-carbethoxyethenyl-3-iodo-7-methoxybenzofuran (0.089 g, $0.152 \mathrm{mmol})$ was dissolved in dry THF $(10 \mathrm{~mL})$ under $\mathrm{N}_{2}$ atmosphere and $\mathrm{LiAlH}_{4}(2.0 \mathrm{M}, 0.38 \mathrm{~mL})$ was slowly added via syringe. The solution was stirred for $24 \mathrm{hr}$ at $50{ }^{\circ} \mathrm{C}$, warmed to room temperature and stirred for $1 \mathrm{hr}$. The reaction was quenched by adding $3 \mathrm{~mL}$ water slowly. After stirring for $20 \mathrm{~min}$, the water-phase was extracted with $\mathrm{CH}_{2} \mathrm{Cl}_{2}(5 \mathrm{~mL} \times 3)$. The organic layer was washed with water and brine, dried over $\mathrm{MgSO}_{4}$, removed by filtration. The filtrate was concentrated in vacuo to give a yellow solid. The solid was chromatographed on silica gel to give a white solid $(0.045 \mathrm{~g}, 55 \%) . R_{f} 0.43$ $($ EtOAc:Hexane $=1: 1) ; \operatorname{mp~} 120-123{ }^{\circ} \mathrm{C} ;{ }^{1} \mathrm{H}$ NMR $(300 \mathrm{MHz}$, $\left.\mathrm{CDCl}_{3}\right) \delta 1.94(2 \mathrm{H}, \mathrm{m}$, propyl C2-H), $2.78(2 \mathrm{H}, \mathrm{t}, J=8.1 \mathrm{~Hz}$, propyl C1-H), $3.70(2 \mathrm{H}, \mathrm{t}, J=6.3 \mathrm{~Hz}$, propyl C3-H), $3.98(3 \mathrm{H}, \mathrm{s})$, $4.02(3 \mathrm{H}, \mathrm{s}), 5.19(2 \mathrm{H}, \mathrm{s}), 6.61(1 \mathrm{H}, \mathrm{s}, \mathrm{C} 3-\mathrm{H}), 6.81(1 \mathrm{H}, \mathrm{s}), 6.91$ $(1 \mathrm{H}, \mathrm{d}, J=8.1 \mathrm{~Hz}), 6.96(1 \mathrm{H}, \mathrm{s}), 7.28-7.46(7 \mathrm{H}, \mathrm{m}) .{ }^{13} \mathrm{C} \mathrm{NMR}$ $\left(75 \mathrm{MHz} \mathrm{CDCl}_{3}\right) \delta 32.8$ (propyl C2-H), 35.0 (propyl C1-H), $56.4\left(\mathrm{OCH}_{3}\right), 56.5\left(\mathrm{OCH}_{3}\right), 62.6\left(\mathrm{OCH}_{2}\right), 71.3\left(\mathrm{OCH}_{2} \mathrm{Ph}\right), 100.6$, $107.4,108.8,112.5,114.1,118.2,124.1,127.5$ (x2), 128.1, 128.8 (x2), 131.3, 137.0, 137.7, 142.7, 144.9, 148.8, 149.9, 156.4.

2-(4-Benzyloxy-3-methoxyphenyl)-5-(3-benzyloxypropyl)6-methoxybenzofuran (19): Yield (92\%). $R_{f} 0.42$ (EtOAc:Hexane $=1: 3) ; \mathrm{mp} 119-121{ }^{\circ} \mathrm{C} ;{ }^{1} \mathrm{HNMR}\left(300 \mathrm{MHz}, \mathrm{CDCl}_{3}\right) \delta 1.94$ $(2 \mathrm{H}, \mathrm{m}), 2.77(2 \mathrm{H}, \mathrm{t}, J=7.2 \mathrm{~Hz}), 3.52(2 \mathrm{H}, \mathrm{t}, J=6.3 \mathrm{~Hz}), 3.86$ $(3 \mathrm{H}, \mathrm{s}), 3.98(3 \mathrm{H}, \mathrm{s}), 4.52(2 \mathrm{H}, \mathrm{s}), 5.20(2 \mathrm{H}, \mathrm{s}), 6.76(1 \mathrm{H}, \mathrm{s}$, C3-H), 6.91 (1H, d, J=8.7 Hz, C4'-H), $7.01(1 \mathrm{H}, \mathrm{s}, \mathrm{C} 7-\mathrm{H})$, $7.23(1 \mathrm{H}, \mathrm{s}), 7.28\left(1 \mathrm{H}, \mathrm{dd}, J=1.8,8.1 \mathrm{~Hz}, \mathrm{C}^{\prime}-\mathrm{H}\right), 7.32-7.40$ $(10 \mathrm{H}, \mathrm{m}), 7.43(1 \mathrm{H}, \mathrm{br} \mathrm{s}) .{ }^{13} \mathrm{C} \mathrm{NMR}\left(75 \mathrm{MHz}, \mathrm{CDCl}_{3}\right) \delta 27.6$, 30.3, 55.9, 56.4, 703, 71.3, 73.1, 94.1, 100.2, 108.3, 114.3, $117.4,120.9,122.0,124.7,126.6,127.5$ (x2), 127.7, 127.9, $128.1,128.5$ (x2), 128.8 (x2), 129.3, 137.1, 138.9, 148.3, 149.9, $154.5,154.8,155.9$.

2-(4-Benzyloxy-3-methoxyphenyl)-6-(3-benzyloxypropyl)5-methoxybenzofuran (20): Yield (95\%). $R_{f} 0.45$ (EtOAc:Hexane $=1: 3) ; \operatorname{mp} 94-97{ }^{\circ} \mathrm{C} ;{ }^{1} \mathrm{H} \mathrm{NMR}\left(300 \mathrm{MHz}, \mathrm{CDCl}_{3}\right) \delta 1.95$ $(2 \mathrm{H}, \mathrm{m}), 2.79(2 \mathrm{H}, \mathrm{t}, J=7.2 \mathrm{~Hz}), 3.51(2 \mathrm{H}, \mathrm{t}, J=6.3 \mathrm{~Hz}), 3.81$ $(3 \mathrm{H}, \mathrm{s}), 3.95(3 \mathrm{H}, \mathrm{s}), 4.49(2 \mathrm{H}, \mathrm{s}), 5.14(2 \mathrm{H}, \mathrm{s}), 6.76(1 \mathrm{H}, \mathrm{s}$, C3-H), 6.87 (1H, d, J=8.4 Hz, C4'-H), $6.90(1 \mathrm{H}, \mathrm{s}, \mathrm{C} 7-\mathrm{H}), 7.25$ $\left(1 \mathrm{H}, \mathrm{dd}, J=1.8,5.4 \mathrm{~Hz}, \mathrm{C} 5{ }^{\prime}-\mathrm{H}\right), 7.27-7.35(10 \mathrm{H}, \mathrm{m}), 7.40-7.42$ $(2 \mathrm{H}, \mathrm{m}) .{ }^{13} \mathrm{C} \mathrm{NMR}\left(75 \mathrm{MHz}, \mathrm{CDCl}_{3}\right) \delta 27.9,30.3,56.1,56.5$, 70.2, 71.3, 73.2, 100.6, 101.3, 108.6, 112.2, 114.3, 117.8, 124.5, $127.5,127.7,127.9(\mathrm{x} 2), 128.0,128.1,128.6,128.8,137.1$, $138.9,148.6,149.6,149.9,154.5,155.9$.

\section{General procedure of debenzylation.}

2-(4-Hydroxy-3-methoxyphenyl)-5-(3-hydroxypropyl)-7methoxybenzofuran (11): To a 2-(4-benzyloxy-3-methoxyphenyl)-5-(3-hydroxypropyl)-7-methoxybenzofuran $(0.05 \mathrm{~g}$, $0.119 \mathrm{mmol})$ in dried $\mathrm{CH}_{2} \mathrm{Cl}_{2}(5 \mathrm{~mL})$ under $\mathrm{N}_{2}$ at $-78{ }^{\circ} \mathrm{C}$ was slowly added via syringe boron tribromide (1.0 $\mathrm{M}_{\text {in }} \mathrm{CH}_{2} \mathrm{Cl}_{2}$, $0.13 \mathrm{~mL}, 1 \mathrm{mmol}$ per benzyloxy group). The solution was stirred for $1 \mathrm{hr}$ at $-78^{\circ} \mathrm{C}$, warmed to room temperature and stirred for $1 \mathrm{hr}$. The reaction was quenched by adding $2 \mathrm{~mL}$ water slowly. After stirring for $20 \mathrm{~min}$, the solvent was evaporated, extracted with EtOAc $(5 \mathrm{~mL} \times 3)$, dried over anhydrous $\mathrm{Na}_{2} \mathrm{SO}_{4}$ and concentrated. The residue was chromatographed on silica gel to give a white solid $(0.034 \mathrm{~g}, 88 \%) . R_{f} 0.21($ EtOAc:Hexane $=$ 1:1); mp $102-104{ }^{\circ} \mathrm{C} ;{ }^{1} \mathrm{H} \mathrm{NMR}\left(300 \mathrm{MHz}, \mathrm{CDCl}_{3}\right) \delta 1.95(2 \mathrm{H}$, $\mathrm{m}$, propyl C2-H), $2.80(2 \mathrm{H}, \mathrm{t}, J=8.1 \mathrm{~Hz}$, propyl C1-H), 3.70 $(2 \mathrm{H}, \mathrm{t}, J=6.3 \mathrm{~Hz}$, propyl C3-H), 3.97 (3H, s, C3'-OMe) 4.01 (3H, C7-OMe), 6.65 (1H, s, C6-H), 6.77 (1H, s, C2'-H), 6.99 $(1 \mathrm{H}, \mathrm{d}, J=7.8 \mathrm{~Hz}, \mathrm{C} 5 '-\mathrm{H}), 7.07(1 \mathrm{H}, \mathrm{s}, \mathrm{C} 3-\mathrm{H}), 7.37(1 \mathrm{H}, \mathrm{dd}$, $\left.J=1.8,8.4 \mathrm{~Hz}, \mathrm{C} 6{ }^{\prime}-\mathrm{H}\right), 7.40(1 \mathrm{H}, \mathrm{d}, J=1.5 \mathrm{~Hz}, \mathrm{C} 4-\mathrm{H}) .{ }^{13} \mathrm{C}$ $\mathrm{NMR}\left(75 \mathrm{MHz}, \mathrm{CDCl}_{3}\right) \delta 32.8$ (propyl C2), 35.0 (propyl C1), $56.3\left(\mathrm{C}^{\prime}-\mathrm{OCH}_{3}\right), 56.4\left(\mathrm{C} 7-\mathrm{OCH}_{3}\right), 62.6\left(\mathrm{CH}_{2} \mathrm{OH}\right), 100.3(\mathrm{C} 3)$, 107.3 (C2'), 107.8 (C6), 112.5 (C4), 115.0 (C6'), 118.9 (C5'), 123.2 (C3a), 131.3 (C1'), 137.6 (C5), 142.5 (C7), 144.9 (C4'), 146.4 (C3'), 146.9 (C7a), $156.6(\mathrm{C} 2)$.

2-(4-Hydroxy-3-methoxyphenyl)-5-(3-hydroxypropyl)-6methoxybenzofuran (12): Yield (82\%). $R_{f} 0.21$ (EtOAc:Hexane $=1: 1) ; \operatorname{mp~} 128-131{ }^{\circ} \mathrm{C} ;{ }^{1} \mathrm{H} \mathrm{NMR}\left(300 \mathrm{MHz} \mathrm{CDCl}_{3}\right) \delta$ $1.87(2 \mathrm{H}, \mathrm{m}$, propyl C2-H), $2.76(2 \mathrm{H}, \mathrm{t}, J=7.2 \mathrm{~Hz}$, propyl $\mathrm{C} 1-\mathrm{H}), 3.62\left(2 \mathrm{H}, \mathrm{t}, J=6.6 \mathrm{~Hz}, \mathrm{CH}_{2} \mathrm{OH}\right), 6.76(1 \mathrm{H}, \mathrm{s}, \mathrm{C} 7-\mathrm{H})$, $6.92\left(1 \mathrm{H}, \mathrm{d}, J=9 \mathrm{~Hz}, \mathrm{C} 5{ }^{\prime}-\mathrm{H}\right), 7.03(1 \mathrm{H}, \mathrm{s}, \mathrm{C} 3-\mathrm{H}), 7.27(1 \mathrm{H}, \mathrm{s}$, C2'-H), 7.29 (1H, dd, $J=1.8,6.6$ Hz, C6'-H), 7.32 (1H, s, C4-H). ${ }^{13} \mathrm{C}$ NMR $\left(75 \mathrm{MHz}, \mathrm{CDCl}_{3}\right) \delta 26.6$ (propyl C2), 33.7 (propyl $\mathrm{C} 1), 56.1\left(\mathrm{C}^{\prime}-\mathrm{OCH}_{3}\right), 56.3\left(\mathrm{C} 6-\mathrm{OCH}_{3}\right), 62.3\left(\mathrm{CH}_{2} \mathrm{OH}\right), 94.2$ (C7), 99.7 (C3), 107.3 (C6'), 114.9 (C2'), 118.3 (C5'), 121.0 (C5), 122.5 (C4), 123.6 (C3a), 126.2 (C1'), 145.9 (C3'), 146.9 (C4), 154.4 (C7a), 155.2 (C6), 155.7 (C2).

2-(4-Hydroxy-3-methoxyphenyl)-6-(3-hydroxypropyl)-5methoxyben zofuran (13): Yield $(90 \%) . R_{f} 0.25$ (EtOAc:Hexane $=1: 1) ; \operatorname{mp~} 136-142{ }^{\circ} \mathrm{C} ;{ }^{1} \mathrm{H} \mathrm{NMR}\left(300 \mathrm{MHz} \mathrm{CDCl}_{3}\right) \delta$ $1.89(2 \mathrm{H}, \mathrm{m}$, propyl C2-H), $2.86(2 \mathrm{H}, \mathrm{t}, J=7.2 \mathrm{~Hz}$, propyl C1-H), $3.62\left(2 \mathrm{H}, \mathrm{t}, J=6 \mathrm{~Hz}, \mathrm{CH}_{2} \mathrm{OH}\right), 3.88\left(3 \mathrm{H}, \mathrm{s}, \mathrm{C}^{\prime}-\mathrm{OCH}_{3}\right), 3.99$ $\left(3 \mathrm{H}, \mathrm{s}, \mathrm{C} 5-\mathrm{OCH}_{3}\right), 6.80(1 \mathrm{H}, \mathrm{d}, J=0.9 \mathrm{~Hz}, \mathrm{C} 5 '-\mathrm{H}), 6.95(1 \mathrm{H}$, d, $\left.J=8.4 \mathrm{~Hz}, \mathrm{C} 6{ }^{\prime}-\mathrm{H}\right), 7.26$ (1H, s, C3-H), 7.27 (1H, s, C4-H), 7.29 (1H, s, C7-H), 7.31 (1H, s, C2'-H). ${ }^{13} \mathrm{C}$ NMR $(75 \mathrm{MHz}$, $\left.\mathrm{CDCl}_{3}\right) \delta 27.0$ (propyl C2), 33.2 (propyl C1), $55.7\left(\mathrm{C}^{\prime}-\mathrm{OCH}_{3}\right.$ ), $55.9\left(\mathrm{C}^{2}-\mathrm{OCH}_{3}\right), 61.7\left(\mathrm{CH}_{2} \mathrm{OH}\right), 99.8(\mathrm{C} 4), 100.9\left(\mathrm{C}^{\prime}\right), 107.6$ (C5'), 111.6(C6), 115.2 (C7), 118.1 (C6'), 122.9 (C3a), 127.4 (C1'), 128.5 (C3), 146.5 (C4'), 147.2 (C3'), 149.2 (C5), 154.1 (C7a), 155.8 (C2).

4-Hydroxy-3-iodo-5-methoxybenzaldehyde (21): To a solution of vanillin $(1.0 \mathrm{~g}, 6.572 \mathrm{mmol})$, iodine $(2.081 \mathrm{~g}, 7.887$ $\mathrm{mmol})$ and silver sulfate $(2.459 \mathrm{~g}, 7.887 \mathrm{mmol})$ in EtOH (50 $\mathrm{mL}$ ) was stirred for $1 \mathrm{hr}$ at room temperature. The solvent was evaporated, extracted with $\mathrm{CH}_{2} \mathrm{Cl}_{2}(5 \mathrm{~mL} \times 3)$, and the organic layer was washed with water $(20 \mathrm{~mL})$ and brine $(20 \mathrm{~mL})$. The combined organic layer was dried over anhydrous $\mathrm{MgSO}_{4}$ and 
concentrated. The residue was chromatographed on silica gel to give a white solid $(1.552 \mathrm{~g}, 85 \%) . R_{f} 0.34$ (EtOAc:Hexane $=$ 1:3); mp $178-181{ }^{\circ} \mathrm{C} ;{ }^{1} \mathrm{H}$ NMR $\left(300 \mathrm{MHz}, \mathrm{CDCl}_{3}\right) \delta 3.97(3 \mathrm{H}$, s, $\left.\mathrm{OCH}_{3}\right), 6.69(1 \mathrm{H}, \mathrm{s}, \mathrm{OH}), 7.36(1 \mathrm{H}, \mathrm{d}, J=1.5 \mathrm{~Hz}, \mathrm{C} 6-\mathrm{H})$, $7.81(1 \mathrm{H}, \mathrm{d}, J=1.5 \mathrm{~Hz}, \mathrm{C} 2-\mathrm{H}), 9.75(1 \mathrm{H}, \mathrm{s}, \mathrm{CHO}) .{ }^{13} \mathrm{C} \mathrm{NMR}$ $\left(75 \mathrm{MHz} \mathrm{CDCl}_{3}\right) \delta 56.8$ (OMe), 80.7 (C3-I), 108.8 (C6), 131.2 (C1), 136.4 (C2), 146.6 (C5), 151.5 (C4), 189.7 (C=O).

2-(4-Benzyloxy-3-methoxyphenyl)-7-methoxybenzofuran5-carbaldehyde (23): To a solution of 4-hydroxy-3-iodo-5methoxybenzaldehyde 21 (0.145 g, $0.523 \mathrm{mmol}), \mathrm{Pd}\left(\mathrm{PPh}_{3}\right)_{4}$ (0.018 g, $0.026 \mathrm{mmol})$, acetylene derivative (22) (0.187 g, $0.784 \mathrm{mmol})$ and $\mathrm{CuI}(0.005 \mathrm{~g}, 0.026 \mathrm{mmol})$ in DMF $(8 \mathrm{~mL})$ under $\mathrm{N}_{2}$ was added $\mathrm{Et}_{3} \mathrm{~N}(0.146 \mathrm{~mL}, 1.046 \mathrm{mmol})$, and stirred for $14 \mathrm{hr}$ at room temperature. The organic-phase was extracted with $\mathrm{CH}_{2} \mathrm{Cl}_{2}(5 \mathrm{~mL} \times 4)$, washed with water several times, and the combined organic layer was dried over anhydrous $\mathrm{MgSO}_{4}$ and concentrated. The residue was chromatographed on silica gel to give a yellow solid $(0.19 \mathrm{~g}, 92 \%) . R_{f} 0.3$ (EtOAc:Hexane $=$ $1: 2) ; \operatorname{mp~} 160-162{ }^{\circ} \mathrm{C} ;{ }^{1} \mathrm{H}$ NMR (300 MHz, $\left.\mathrm{CDCl}_{3}\right) \delta 3.98$ $\left(3 \mathrm{H}, \mathrm{s}, \mathrm{C} 3\right.$ ' $\left.-\mathrm{OCH}_{3}\right), 4.06\left(3 \mathrm{H}, \mathrm{s}, \mathrm{C} 7-\mathrm{OCH}_{3}\right), 5.19(2 \mathrm{H}, \mathrm{s}), 6.91$ (1H, s, C3-H), 6.93 (1H, s, C2'-H), 7.29-7.45 (8H, m), 7.65 $(1 \mathrm{H}, \mathrm{s}, \mathrm{C} 4-\mathrm{H}), 9.96(1 \mathrm{H}, \mathrm{s}, \mathrm{CHO}) .{ }^{13} \mathrm{C} \mathrm{NMR}\left(75 \mathrm{MHz}, \mathrm{CDCl}_{3}\right)$ $\delta 56.5(\mathrm{x} 2), 71.2,100.9,104.5,108.9,114.0,118.5,119.2,123.1$, $127.5,128.2,128.8,131.2,133.6,136.8,146.0,147.6,149.3$, $149.9,158.0,191.9(\mathrm{C}=\mathrm{O})$.

2-(4-Benzyloxy-3-methoxyphenyl)-5-(carbethoxyethenyl)7-methoxybenzofuran (24): To a solution of 2-(4-benzyloxy3-methoxyphenyl)-7-methoxybenzofuran-5-carbaldehyde $\mathbf{2 3}$ $(0.270 \mathrm{~g}, 0.75 \mathrm{mmol})$ in $\mathrm{CH}_{2} \mathrm{Cl}_{2}(20 \mathrm{~mL})$ under $\mathrm{N}_{2}$ was added (carbethoxymethylene)triphenylphosphorane $(0.264 \mathrm{~g}, 0.99$ $\mathrm{mmol}$ ). The reaction mixture was refluxed for $6 \mathrm{hr}$, and warmed to room temperature. The solution was extracted with $\mathrm{CH}_{2} \mathrm{Cl}_{2}$ $(5 \mathrm{~mL} \times 3)$, washed with water $(20 \mathrm{~mL})$ and brine $(20 \mathrm{~mL})$, dried over anhydrous $\mathrm{MgSO}_{4}$, and concentrated. The residue was chromatographed on silica gel to give a white solid $(0.341 \mathrm{~g}$, 99\%). $R_{f} 0.2$ (EtOAc:Hexane = 1:4); $\mathrm{mp} 142-144{ }^{\circ} \mathrm{C} ;{ }^{1} \mathrm{H}$ NMR (300 MHz, acetone- $\left.d_{6}\right) \delta 1.28\left(3 \mathrm{H}, \mathrm{t}, J=7.1 \mathrm{~Hz}, \mathrm{CH}_{3}\right), 3.92$ $(3 \mathrm{H}, \mathrm{s}), 4.07(3 \mathrm{H}, \mathrm{s}), 4.20\left(2 \mathrm{H}, \mathrm{q}, J=7.1 \mathrm{~Hz}, \mathrm{OCH}_{2}\right), 5.15(2 \mathrm{H}$, s), $6.52(1 \mathrm{H}, \mathrm{d}, J=16.0 \mathrm{~Hz}$, trans ethenyl C1-H), $6.52(1 \mathrm{H}, \mathrm{d}$, $J=16 \mathrm{~Hz}), 7.12(1 \mathrm{H}, \mathrm{d}, J=8.3 \mathrm{~Hz}), 7.15(1 \mathrm{H}, \mathrm{s}), 7.26(1 \mathrm{H}, \mathrm{d}$, $J=1.4 \mathrm{~Hz}), 7.31-7.52(8 \mathrm{H}, \mathrm{m}), 7.72(1 \mathrm{H}, \mathrm{d}, J=16.0 \mathrm{~Hz}$, trans ethenyl C2-H). ${ }^{13} \mathrm{C}$ NMR $\left(75 \mathrm{MHz}\right.$, acetone- $\left.d_{6}\right) \delta 14.8\left(\mathrm{CH}_{3}\right)$, $56.5\left(\mathrm{OCH}_{3}\right), 56.7\left(\mathrm{OCH}_{3}\right), 60.8\left(\mathrm{OCH}_{2}\right), 71.5\left(\mathrm{OCH}_{2}\right), 101.7$, 106.5, 109.8, 115.1, 118.1 (trans ethenyl-C1), 118.8, 124.3, $128.7,128.9,129.4,131.9,132.5,138.4,146.1$ (trans ethenylC2), 146.1, 146.6, 150.5, 151.3, 158.1, $167.4(\mathrm{C}=\mathrm{O})$.

2-(4-Hydroxy-3-methoxyphenyl)-5-(carbethoxyethenyl)-7methoxybenzofuran (25): To a solution of 2-(4-benzyloxy3-methoxyphenyl)-5-(carbethoxyethenyl)-7-methoxybenzofuran $24(0.29 \mathrm{~g}, 0.63 \mathrm{mmol})$ in $\mathrm{CH}_{2} \mathrm{Cl}_{2}(25 \mathrm{~mL})$ was added $\mathrm{TiCl}_{4}\left(0.7 \mathrm{~mL}, 0.7 \mathrm{mmol}, 1.0 \mathrm{M}\right.$ in $\left.\mathrm{CH}_{2} \mathrm{Cl}_{2}\right)$ dropwise at ambient temperature. The reaction was monitored by TLC and quenched by treatment with $\mathrm{MeOH}$. The solvent was removed and the residue was chromatographed on silica gel to give a white solid $(0.228 \mathrm{~g}, 98 \%) R_{f} 0.5$ (EtOAc:Hexane $\left.=1: 2\right) ; \mathrm{mp} 149-151{ }^{\circ} \mathrm{C}$; ${ }^{1} \mathrm{H}$ NMR $\left(300 \mathrm{MHz}\right.$, acetone- $\left.d_{6}\right) \delta 1.27\left(3 \mathrm{H}, \mathrm{t}, J=7.1 \mathrm{~Hz}, \mathrm{CH}_{3}\right)$, $3.91(3 \mathrm{H}, \mathrm{s}), 4.07(3 \mathrm{H}, \mathrm{s}), 4.20\left(2 \mathrm{H}, \mathrm{q}, J=7.1 \mathrm{~Hz}, \mathrm{OCH}_{2}\right), 6.52$ $(1 \mathrm{H}, \mathrm{d}, J=16.0 \mathrm{~Hz}$, trans ethenyl C1-H), $6.94(1 \mathrm{H}, \mathrm{d}, J=8.2$
$\mathrm{Hz}), 7.07(1 \mathrm{H}, \mathrm{s}), 7.24(1 \mathrm{H}, \mathrm{d}, J=1.4 \mathrm{~Hz}), 7.41(1 \mathrm{H}, \mathrm{dd}, J=8.2$, $2.0 \mathrm{~Hz}), 7.70(1 \mathrm{H}, \mathrm{d}, J=16.0 \mathrm{~Hz}$, trans ethenyl C2-H), $8.07(1 \mathrm{H}$, s). ${ }^{13} \mathrm{C} \mathrm{NMR}\left(75 \mathrm{MHz}\right.$, acetone- $\left.d_{6}\right) \delta 14.8\left(\mathrm{CH}_{3}\right), 56.6\left(\mathrm{OCH}_{3}\right)$, $56.6\left(\mathrm{OCH}_{3}\right), 60.9\left(\mathrm{OCH}_{2}\right), 100.1,106.5,109.4,115.5,116.6$ (trans ethenyl-C1), 118.0, 119.5, 123.0, 129.5, 131.9, 132.6, 146.1 (trans ethenyl-C2), 146.6, $148.9(\mathrm{x} 2), 158.4,167.5(\mathrm{C}=\mathrm{O})$.

Ailanthoidol (14): To a solution of 2-(4-hydroxy-3-methoxyphenyl)-5-(carbethoxyethenyl)-7-methoxy benzofuran $25(0.170 \mathrm{~g}, 0.46 \mathrm{mmol})$ in THF $(10 \mathrm{~mL})$ was added a solution

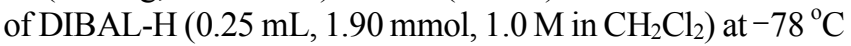
under $\mathrm{N}_{2}$ atmosphere. After being stirred at the same conditions for $2 \mathrm{hr}$, the reaction was quenched with saturated $\mathrm{Na}_{2} \mathrm{CO}_{3}$. $10 \mathrm{H}_{2} \mathrm{O}(5 \mathrm{~mL})$, and the resulting mixture was partitioned between $\mathrm{CH}_{2} \mathrm{Cl}_{2}(50 \mathrm{~mL})$ and water $(30 \mathrm{~mL})$. The organic layer was washed with water $(20 \mathrm{~mL})$ and brine $(20 \mathrm{~mL})$, dried over anhydrous $\mathrm{MgSO}_{4}$ and concentrated. The residue was chromatographed on silica gel to give a white solid $(0.143 \mathrm{~g}, 95 \%) . R_{f} 0.6$ (EtOAc:Hexane = 3:1); mp $199-201{ }^{\circ} \mathrm{C} ;{ }^{1} \mathrm{H} \mathrm{NMR}(300 \mathrm{MHz}$, DMSO-d $\left.d_{6}\right) \delta 3.87\left(3 \mathrm{H}, \mathrm{s}, \mathrm{C} 7-\mathrm{OCH}_{3}\right), 3.93\left(3 \mathrm{H}, \mathrm{s}, \mathrm{C} 3^{\prime}-\mathrm{OCH}_{3}\right)$, $4.13\left(2 \mathrm{H}, \mathrm{d}, J=4.7 \mathrm{~Hz}, \mathrm{CH}_{2} \mathrm{OH}\right), 5.59(1 \mathrm{H}, \mathrm{s}), 6.31(1 \mathrm{H}, \mathrm{dt}, J=$ $5.0,15.8 \mathrm{~Hz}$, trans ethenyl C2-H), $6.57(1 \mathrm{H}, \mathrm{d}, J=15.8 \mathrm{~Hz}$, trans ethenyl C1-H), $6.86\left(1 \mathrm{H}, \mathrm{d}, J=8.2 \mathrm{~Hz}, \mathrm{C} 5{ }^{\prime}-\mathrm{H}\right), 6.90(1 \mathrm{H}$, s, C2'-H), $7.03(1 \mathrm{H}, \mathrm{d}, J=1.4 \mathrm{~Hz}, \mathrm{C} 3-\mathrm{H}), 7.10(1 \mathrm{H}, \mathrm{s}), 7.29(1 \mathrm{H}$, $\left.\mathrm{dd}, J=1.4,8.2 \mathrm{~Hz}, \mathrm{C} 6{ }^{\prime}-\mathrm{H}\right), 7.33(1 \mathrm{H}, \mathrm{s}, \mathrm{C} 4-\mathrm{H}) .{ }^{13} \mathrm{C} \mathrm{NMR}(75$ $\left.\mathrm{MHz}, \mathrm{DMSO}-d_{6}\right) \delta 55.7\left(\mathrm{C}^{\prime}-\mathrm{OCH}_{3}, \mathrm{C} 7-\mathrm{OCH}_{3}\right), 61.6\left(\mathrm{CH}_{2} \mathrm{OH}\right)$, 100.1 (C3), 104.3 (C4), 108.6 (C6), 110.8 (C2'), 115.8 (C6'), 117.8 (C5'), 121.0 (trans ethenyl C2), $128.9\left(\mathrm{Cl}^{\prime}\right), 129.5$ (C3a), 130.7 (trans ethenyl C1), 133.1 (C5), 142.3 (C7), 144.5 (C4'), 147.5 (C3'), 147.8 (C7a), 156.1 (C2).

Cell culture and cell viability assay. RAW264.7 murine macrophages were obtained from the Korean Cell Bank (Seoul, Korea) and cultured in DMEM containing 10\% FBS, $100 \mathrm{U} / \mathrm{mL}$ penicillin, and $100 \mu \mathrm{g} / \mathrm{mL}$ streptomycin at $37{ }^{\circ} \mathrm{C}$ in $5 \% \mathrm{CO}_{2}$. The effects of the prepared 13 benzofurans 2-14 on cell viability were tested using the CellTiter $96^{\circledR}$ AQueous One Solution Assay of cell proliferation (Promega, Madison, WI), which uses colorimetry to count the number of viable cells. This assay was used to determine the number of viable cells remaining after the culturing process was complete. RAW264.7 cells were plated at a density of $2 \times 10^{4}$ cells in a 96-well flat-bottom plate, and the prepared 13 benzofurans 2-14 were added to each plate at concentrations of 0,1 and $10 \mu \mathrm{M}$. After a $24 \mathrm{~h}$ incubation period, the number of viable cells was counted according to the manufacturer's instructions.

Measurement of NO. The amount of NO produced by the mouse macrophage was indicated by the amount that was measured in the RAW264.7 cell culture supernatant. RAW264.7 cells were plated at a density of $5 \times 10^{5}$ cells in a 24 -well cell culture plate with $500 \mu \mathrm{L}$ of culture medium and incubated for $12 \mathrm{~h}$. They were then treated with 1 or $10 \mu \mathrm{M}$ of each compound in $1 \mu \mathrm{g} / \mathrm{mL}$ of LPS and incubated for another $18 \mathrm{~h}$. The amount of NO produced was measured using the Griess reagent system (Promega).

Acknowledgments. This research was financially supported by the Ministry of Education, Science Technology (MEST) and Korea Industrial Technology Foundation (KOTEF) through the Human Resource Training Project for Regional Innovation 
(4R09-0301-003-S000100), and by Priority Research Centers Program through the National Research Foundation of Korea (NRF) funded by the Ministry of Education, Science and Technology (2009-0094072).

\section{References}

1. Katritzky, A. R.; Rees, C. W.; Scriven, E. F. V. Comprehensive Heterocyclic Chemistry II; Pergamon Press: Oxford, UK, 1996; Vol. 2, pp 259-321.

2. Navarro, E.; Alanso, S. J.; Trujillo, J.; Jorge, E.; Perez, C. J. Nat. Prod. 2001, 64, 134.

3. Ikeda, R.; Nagao, T.; Okabe, H.; Nakano, Y.; Matsunaga, H.; Katano, M.; Mori, M. Chem. Pharm. Bull. 1998, 46, 871.

4. Day, S. H.; Chiu, N. Y.; Tsao, L. T.; Wang, J. P.; Lin, C. N. J. Nat. Prod. 2000, 63, 1560 .

5. Craigo, J.; Callahan, M.; Huang, R. C. C.; DeLucia, A. L. Antiviral Res. 2000, 47, 19

6. Zacchino, S.; Rodriguez, G.; Pezzenati, G.; Orellana, G.; Enriz, R.; Gonzalez, S. M. J. Nat. Prod. 1997, 60, 659.

7. Gordaliza, M.; Castro, M.; del Corral, J. M.; Lopez-Vazquez, M.; Feliciano, A. S.; Faircloth, G. T. Bioorg. Med. Chem. Lett. 1997, 7, 2781.

8. Chen, C. C.; Hsin, W. C.; Ko, F. N.; Huang, Y. L.; Ou, J. C.; Teng, C. M. J. Nat. Prod. 1996, 59, 1149.

9. Silva, D. H. S.; Pereira, F. C.; Zanoni, M. V. B.; Yoshida, M.
Phytochemistry 2001, 57, 437.

10. Ward, R. S. Nat. Prod. Rep. 1995, 12, 183.

11. Brader, G.; Greger, H.; Bacher, M.; Kalchhauser, H.; Hofer, O.; Vajrodaya, S. J. Nat. Prod. 1998, 61, 1482.

12. (a) Yue, D.; Yao, T.; Larock, R. C. J. Org. Chem. 2005, 70, 10292. (b) Lin, S.-Y.; Chen, C.-L.; Lee, Y.-J. J. Org. Chem. 2003, 68, 2968. (c) Kao, C.-L.; Chern, J.-W. J. Org. Chem. 2002, 67, 6772. (d) Katritzky, A. R.; Ji, Y.; Fang, Y.; Prakash, I. J. Org. Chem. 2001, 66, 5613. (e) Lutjens, H.; Scammells, P. J. Tetrahedron Lett. 1998, 39,6581 .

13. Choi, D. H.; Hwang, J. W.; Lee, H. S.; Yang, D. M.; Jun, J.-G. Bull. Korean Chem. Soc. 2008, 29, 1594.

14. Chang, H. M.; Cheng, K. P.; Choang, T. F.; Chow, H. F.; Chui, K. Y.; Hon, P. M.; Tan, F. W. L.; Yang, Y.; Zhong, Z. P. J. Org. Chem. $1990,55,3537$.

15. Bang, H. B.; Han S. Y.; Choi, D. H.; Yang D. M.; Hwang, J. W.; Lee, H. S.; Jun, J.-G. Synth. Commun. 2009, 39, 506.

16. Bang, H. B.; Han S. Y.; Choi, D. H.; Hwang, J. W.; Jun, J.-G. Arkivoc 2009, (ii), 112.

17. Sheen, W.-S.; Tsai, I.-L.; Teng, C.-M.; Chen, I.-S. Phytochemistry 1994, 36, 213.

18. (a) Vernooy, J. H.; Dentener, M. A.; Suylen, R. J. van.; Buurman, W. A.; Wouters, E. F. Am. J. Respir Cell Mol. Biol. 2002, 26, 152. (b) Sakagami, T.; Vella, J.; Dixon, M. F.; Rourke, J.; Radcliff, F.; Sutton, P.; Shimoyama, T.; Beagley, K.; Lee, A. Infect. Immun. 1997, 65, 3310 .

19. Kwon, K. H.; Murakami, A.; Hayashi, R.; Ohigashi, H. Biochem. Biophys. Res. Commun. 2005, 337, 647. 